\title{
Prostate cancer detection improved by optimization of biopsy strategy: Bayesian framework of Network Meta -Analysis and HSROC model for diagnostic accuracy
}

Ilham Akbar Rahman ( $\nabla$ ilhamakbaarr@gmail.com )

Universitas Hasanuddin Fakultas Kedokteran https://orcid.org/0000-0002-9469-1943

Ilham Fauzan

Universitas Hasanuddin Fakultas Kedokteran

Syakri Syahrir

Universitas Hasanuddin Fakultas Kedokteran

Harry Nusaly

Universitas Hasanuddin Fakultas Kedokteran

Firdaus Kasim

Universitas Hasanuddin Fakultas Kedokteran

\section{Research}

Keywords: Prostate cancer, prostate biopsy, transrectal prostate biopsy, fusion prostate biopsy, systematic prostate biopsy

Posted Date: April 28th, 2020

DOI: https://doi.org/10.21203/rs.3.rs-24206/v1

License: (c) (i) This work is licensed under a Creative Commons Attribution 4.0 International License.

Read Full License 


\section{Abstract}

Background: The increasing problem in prostate cancer is associated with over-diagnosis and overtreatment. The transrectal ultrasonography (TRUS)-guided biopsy as a current gold standard investigation poses low positive detection rate causing unnecessary biopsies. The choice of optimal biopsy strategy needs to be completely defined. Therefore, in the absence of direct statistical comparison and limited evidence, we undertook the advantage of Bayesian network meta-analysis and Bayesian prediction in the HSROC model to present a method for optimizing biopsy strategy in prostate cancer.

Materials and Methods: Relevant 24 studies were comprehensively retrieved through online databases of EMBASE, MEDLINE, and CENTRAL up to February 2020. Markov chain Monte Carlo simulation and SUCRA were used to calculate the rank probability using odds ratio (OR) with $95 \% \mathrm{Cl}$. HSROC model was used to formulate the predicted true sensitivity and specificity of each biopsy strategy. Data analysis was performed using STATA, R, and WinBUGS statistics.

Results: Six different PCa biopsy strategies based on four outcomes were ultimately analyzed in this study with a total of 6768 patients. The rank probability showed that the overall PCa detection rate was higher in FUS+TRUS-GB, MRI-GB, and FUS-GB. In terms of clinically significant PCa detection, FUS+TRUS$G B$, and FUS-GB had a relatively higher clinically significant detection rate. Moreover, MRI-GB, FUS-GB, and FUS+TRUS-GB were less associated with insignificant PCa detection rate. MRI-GB and FUS-GB had the highest predicted of true sensitivity and specificity.

Conclusion: Taken together, in comprehensive consideration of four endpoints, our analysis highlights the important finding that FUS+TRUS-GB, FUS-GB, and MRI-GB showed their superiority compared with other strategies. TRUS-GB was more associated with over-diagnosis of insignificant PCa, lower sensitivity, and specificity.

\section{Background}

Prostate cancer (PCa) with 1.1 million estimated new cases in 2012 is the second worldwide most prevalently diagnosed malignancy in male (1). It is the second most frequent cancer and the fifth leading cause of cancer death in men (2). In Indonesia, prostate cancer is the third most common urologic cancer in men according to the GLOBOCAN 2012 study (3).

Currently, the worldwide usage of the triad diagnostic strategy of PCa consisting serum prostate-specific antigen (PSA) measurement, abnormal digital rectal examination (DRE) and transrectal ultrasoundguided biopsies (TRUS-GB) had improved the detection rate of early PCa (4). The European Association of Urology, the US Preventive Services Task Force, and the UK National Institute for Health and Care Excellence suggest transrectal ultrasonography (TRUS) - guided biopsy as a gold standard investigation in the diagnosis of PCa (5). However, recent previous studies showed that this biopsy protocol resulted in a positive detection rate of only $17-36 \%,(6,7)$ with low sensitivity of $27 \%-40.3 \%$ which could easily carry a high rate of missed cancer (8). The crucial dilemma was being encountered by the physicians to decide 
whether to treat the patient or not in initial set of negative prostate biopsies. Because prostate cancer is often multifocal and the prostate volume taken by the standard sextant biopsy technique is relatively small, the possibility persists that these individuals may have cancer despite an initial set of negative biopsies and that a significant number of patients (13-41\%) with persistently elevated PSA after an initial negative biopsy had a positive repeat biopsy leading to the conclusion that this method is associated with underdetection of high-grade prostate cancers and overdetection of low-grade cancers. It has been suggested that ideal systematic biopsy strategy remained to be completely defined (9-12).

With the problem of PCa's over-diagnosis and over-treatment, several imaging-guided biopsy strategies had been utilized in an effort to increase the prostate cancer detection rate (13). It was difficult for physicians to compare and provide the optimal biopsy strategy due to the absence of direct head to head statistical comparison analysis and limited evidence. Therefore, we undertook the advantage of network meta-analysis and anticipated it to provide a hierarchy of diverse methods in a wide spectrum of the population $(14,15)$. Six different PCa biopsy strategies consisted of TRUS-GB, TPUS-GB, CEUS-GB, MRIGB, FUS-GB, FUS-GB plus TRUS-GB, and four clinical outcomes composed of overall PCa detection, significant $\mathrm{PCa}$ detection, insignificant PCa detection as well as hierarchical summary receiver operating characteristics, were fundamentally analyzed in this study. As a result, our analysis was expected to provide some references for daily clinical practice.

\section{Methods}

Literature search strategy and study selection

Eligible articles were extracted from online databases including EMBASE, MEDLINE, and CENTRAL up to February 2020. The search strategy included two parts (prostate cancer and biopsy strategy) using certain keywords in combination with Medical Subject Headings (MeSH) terms and words: "prostate cancer", "biopsy strategy”, "targeted biopsy", "systematic biopsy", "TRUS-GB”, “TPUS-GB”, “FUS-GB”, "FUS+TRUS-GB", "CEUS-GB", "MRI-GB". Full texts and abstracts were initially and independently screened by 2 reviewers. Full texts and abstracts were assessed according to inclusion and exclusion criteria. Insignificant studies were excluded. Discrepancies between 2 reviewers were settled in a discussion in third reviewer involvement. Ethical approval was not required because it did not contain individual patient's data.

Data extraction and quality assessment

Studies included in this articles should meet the following criteria: (1) Subjects were patients with prostate cancer; (2) The required data to formulate NMA and HSROC were available; (3) The comparison should be at least two different biopsy strategy; (4) The article language was published in English; (5) Studies should be in RCTs and original study. Two reviewers (IAR and AT) were individually extracted and reviewed data based on study selection criteria using standardized, structured and piloted extraction forms. The results were checked and discussed by IAR and AT to finalize the included studies. Any discrepancies were resolved in discussion with third reviewer involvement. For each included study, 
important informations were extracted including author's name, publication year, number of sample sizes, mean age, prostate volume, mean PSA level, study design, intervention, overall PCa detection rate, clinical significant PCa detection rate, insignificant PCa detection rate, true positive, false positive, false negative and true negative. If the required data could not be directly acquired from articles, it was manually calculated using available data according to studies $(16,17)$. Table 2 comprehensively demonstrated all of the abovementioned data. Cochrane Collaboration's Risk of Bias tool was used to assess the appropriateness of the included studies and the strength of the evidence. The potential source risk of bias was arisen from (1) Random sequence generation; (2) Allocation concealment; (3) Blinding of participants and personnel; (4) Blinding of outcome assessment; (5) Incomplete outcome data; (6) Selective reporting; (7) Other bias. Low, high or unclear risk of bias was used for judgments. Figure 1 showed the detailed literature search and selection process. Publication bias was examined by Begg's and Egger's tests (18).

\section{Outcomes}

Overall prostate cancer detection rate, clinically significant prostate cancer detection rate, insignificant prostate cancer detection rate, and Hierarchical Summary Receiver Operating Characteristic (HSROC) were ultimately analyzed as endpoints in this study. Clinically significant prostate cancer (CSPCA) was defined as prostate cancer that embodies of minimum Gleason score of 7 whereas insignificant prostate cancer was defined as below or equal to Gleason score of 6 .

Statistical Analysis

We performed a Bayesian network meta-analysis model for each outcome separately, (19) combining direct evidence for each comparison (eg, from studies comparing interventions $A$ with $B$ ) with indirect evidence (eg, from studies comparing $A$ with $C$ and studies comparing $B$ with $C$ ). Network plots were generated to demonstrate the comparison scheme for each PCa biopsy strategies using Stata software (version 12.0; StataCorp LP, College Station, TX). The probability of each biopsy strategy is superior to all others, second best, the third best and so on, was calculated using MCMC simulation and Surface Under the Cumulative Ranking curve (SUCRA). The biopsy strategy then to be determined as the best based on consideration from MCMC simulation and SUCRA. For Markov chain Monte Carlo simulation, a Bayesian framework in network meta-analysis consisting of multiple treatments was conducted using $\mathrm{R}$ language (version 1.2.1335) and WinBUGS (MRC Biostatistics Unit, Cambridge, UK). The simulation was based on 40000 iterations with a burn-in of 10000 iterations. For SUCRA value, summary numerical value was calculated. If SUCRA value would be $100 \%$, the intervention is certainly the best and if SUCRA value would be $0 \%$, the intervention is certainly the worst. Odds ratio (OR) with $95 \%$ confidence interval $(\mathrm{Cl})$ was used for each intervention. Random effect model was preferred instead of fixed effect model based on our model fit assessment. Also, inconsistency was not found on all three outcome analysis.

\section{Results}

Search results and included strategies 
A total of 248 citations retrieved by search strategy were included in this network meta-analysis study. Then, full-text screen was conducted and 86 studies were excluded because of reviews, case report, duplicates, and non-English language. Thirty-two articles were excluded after titles and abstract reading. Thirteen articles were removed after full-text review. Finally, 24 (17,18, 19, 20-26, 27-36,37-40,) studies fulfilled the inclusion criteria consisting of a total of 6768 patients were eligible and added for further analysis. All included studies were RCTs. Several different strategies consisted of TRUS-GB, MRI-GB, MRI+TRUS (FUS)-GB, FUS+TRUS-GB, CEUS-GB, TPUS-GB. Four endpoints were ultimately analyzed including overall PCa detection, clinically significant PCa detection, insignificant PCa detection, and HSROC. The flow chart of study search and selection procedure was shown in Figure 1. The network structure graph was shown in Figure 2.

Overall PCa detection rate

The results of overall PCa detection rate were analyzed by calculating 17 studies consisting of 6 biopsy strategies including TRUS-GB, FUS + TRUS -GB, MRI+TRUS (FUS)-GB, MRI-GB, TPUS-GB, CEUS-GB, and network structure diagrams were shown in Figure 2A. The comparison of efficacy between different biopsy strategies for OR and 95\% confidence interval was presented in league table Figure 3A. As shown in the result with TRUS-GB as comparator, FUS + TRUS-GB (RR: 1.35, 95\% Crl: $0.8-2.2$ ) was slightly better than MRI-GB (RR: 1.28, 95\% Crl: 0.9 - 1.78), and FUS-GB (RR: 1.23, 95\% Crl: 0.93 - 1.61). The cumulative rank probability based on SUCRA value showed that the biopsy strategies from best to worst in terms of overall PCa detection rate were FUS + TRUS-GB, MRI-GB, MRI+TRUS (FUS)-GB, TPUS-GB, CEUS-GB, and TRUS-GB. Figure 4A was a cumulative rank plot with the surface under the cumulative rank curve (SUCRA) of each strategy and its detailed ranking values were summarized in Table 3A.

Clinically significant PCa detection rate

Fourteen studies were contributed to the analysis of clinically significant PCa detection rate. Figure 2B presented the network structure diagrams. The efficacy of each biopsy strategy was compared to each other and was served in league table Figure 3B. Our analysis showed that in the case of TRUS-GB as reference, FUS -GB (RR: 1.51, 95\% Crl: 0.87 - 2.61) was better than FUS + TRUS GB (RR: 1.47, 95\% Crl: 0.55 - 3.89), TPUS-GB (RR: $1.2,95 \%$ Crl: $0.23-6.1$ ) and MRI-GB (RR: $1.09,95 \%$ Crl: $0.56-2.15$ ). As indicated by the results of ranking analysis based on SUCRA which is shown in figure 4B and table 3B, FUS-GB, FUS+TRUS-GB, TPUS-GB, MRI-GB, TRUS-GB, and CEUS-GB were ranked from best to worst respectively.

Insignificant PCa detection rate

The efficacy of each biopsy strategy in terms of insignificant PCa detection was also analyzed. Fourteen trials were used to analyze this endpoint. The detailed comparison was served in network structure diagrams in Figure 2C. Moreover, the efficacy of different biopsy strategies was compared to each other and was shown in league table Figure $3 \mathrm{C}$. It is obviously found that for TRUS-GB as comparator, CEUS-GB (RR: 1.3, 95\% Crl: 0.50 - 3.63) and TPUS-GB (RR: 1.11, 95\% Crl: 0.22 - 5.52) were more associated with 
insignificant PCa detection compared to MRI-GB (RR: 0.67, 95\% Cl: 0.33 - 1.32) and FUS-GB (RR: 0.71, $95 \% \mathrm{Crl}: 0.39$ - 1.24) which were more less associated with insignificant PCa detection. The results of SUCRA rank probability Figure $4 \mathrm{C}$ sorting from more associated to less associated with insignificant PCa detection were CEUS-GB, TRUS-GB, TPUS-GB, FUS+TRUS-GB, FUS-GB, and MRI-GB. The detailed SUCRA values were shown in Table 3C.

HSROC

Based on data extraction, it showed that a large heterogeneity in the reporting of diagnostic accuracy measures. For such reasons, the average operating points (summary of sensitivity and specificity) with the corresponding $95 \% \mathrm{Cl}$ were computed using the summary Receiver operating characteristic (ROC) curves using the hierarchical model proposed by Rutter and Gatsonis (44). Due to limited primary data, only 4 HSROC curves consisting TRUS-GB, FUS-GB, MRI-GB, and CEUS-GB were managed to be analyzed. It is easily found that MRI-GB and FUS-GB presented the highest predicted sensitivity and specificity for overall PCa detection. Figure 5 summarized the HSROC curve.

\section{Discussion}

Although the gold standard examination for PCa was TRUS-GB, we were still faced with so many problems of over-diagnosis and over-treatment in PCa leaving confusion in both clinicians and patients to define the optimal biopsy strategy (45). Therefore, another biopsy strategy had gained more and more interests. Hence, this study was conducted to clarify this question and It was anticipated to provide some references for clinical work.

To the best of our knowledge, this is the first largest study to combine Bayesian framework in network meta-analysis and HSROC model in biopsy strategy for prostate cancer detection. Even in the case of limited primary evidence, all relevant evidences of biopsy strategy in prostate cancer patients were able to be integrated simultaneously by performing network meta-analysis. Moreover, to ensure the firm conclusion, we analyzed the sensitivity and specificity of biopsy strategy in the form of Bayesian prediction in hierarchical summary receiver operating characteristic. Bayesian framework was used to enhance the quality of our analysis so that it could provide the results with more confidence for decision making $(46,47)$.

This study systematically evaluated the comparative efficacy of different biopsy strategies for patients with PCa. A total of 6768 cases from 24 studies were ultimately analyzed in our study. As illustrated by the results, in terms of overall Pca and clinically significant PCa detection rate outcome, FUS + TRUS-GB, MRI-GB, and MRI+TRUS (FUS)-GB were ranked as the best. As for TRUS-GB, it was more associated with lower overall PCa detection and was more inferior in clinically significant PCa detection. Previous study has shown that the overall PCa detection rate for the FUS+TRUS-GB was $56 \%$ compared with FUS-GB alone and TRUS-GB alone (39\% and 7.7\% respectively). This highlights that the additional TRUS-GB in FUS-GB yielded an absolute 10.4\% increase in overall PCa detection (32). In a trial involving 648 patients, FUS-GB detected $14.3 \%$ of cases in prostate cancer that was missed/undetected by systematic 12 core 
biopsy of which $86.7 \%$ were considered to be high risk tumor (20). FUS-GB was also as effective as 12 core-RB in detecting cSPCA which FUS-GB managed to detect $97 \%$ csPCa with a PIRADS score of 4-5 indicating that 12-core RB may be replaced by two-core FUS-GB especially in disease with PIRADS scores of 4-5 (37). This explained how the FUS-GB was superior than other treatments. Compared with 6-core TRUS-GB and 12 core TRUS-GB, the MRI-GB had significantly lower (7\%) of low-grade PCa rate showing that a screening strategy with MRI-GB could significantly decrease over-diagnosis of low-grade disease. Moreover, MRI-GB strategy would result in a significant decrease of biopsies as more than two-third of screened men with PSA level more than $\geq 3.0 \mathrm{ng} / \mathrm{ml}$ possess a negative MRI (48). Low-grade PCa rate of MRI-GB was significantly lower when compared to 6-core TRUS-GB (17\%) and 12-core TRUS-GB (28\%), showing that a screening strategy combined with MRI-GB could significantly yield the reduction of overdiagnosis in low-grade disease (21). These study observations also were in line with our results which proved that MRI-GB, FUS-GB, and FUS+TRUS-GB were less associated with insignificant PCa detection rate. Furthermore, from HSROC analysis, MRI and FUS guided biopsy resulted for the highest sensitivity and specificity for PCa detection.

As for TRUS-GB, TPUS-GB, and CEUS-GB, they were ranked lower compared to others in all four endpoints. Although CEUS-GB provided a statistically significant improvement in discrimination between benign and malignant biopsy sites, it also had relatively low ROC areas thus may not be sufficient to predict which patients have benign versus malignant disease (38). As for TPUS-GB, the limitations of TPUS-GB lied in its difficulties in visualizing hypoechoic areas thus may be the reason for low sensitivity rate (49).

The surgeons were given the opportunity to perform biopsy directly to the suspected areas rather than randomly through the appearance of new biopsy technology such as FUS-GB, MRI-GB, and FUS+TRUS-GB $(28,29)$. Even in the good sense that it is rational to conclude that the more cores, the higher PCa detection rate. However, an interesting fact was found that targeted biopsy could result in a significantly higher rate for detecting PCa compared to random biopsy and therefore demand fewer cores (32). In the era of over-diagnosis and over-treatment of PCa, all these reasoning could lead to the potential of targeted biopsy in improving benefit-to-harm ratio (40). The benefit of a mortality reduction achieved by screening must be balanced against the harms of unnecessary biopsies and overdiagnosis, particularly in elderly men $(52,53)$. This benefit is seen in terms of greater detection of csPCa and a reduction in number of biopsy cores received during a biopsy procedure, showing the capacity to prevent unnecessary biopsies and possibly lowering the overall burden of adverse effects from the invasive biopsy procedure. This result leads us to the reasoning that the potential of MRI-GB, FUS-GB, and FUS+TRUS-GB could become as the first-line technique for detecting PCa in upcoming years.

There are several main strengths of this review. Firstly, the inclusion criteria were limited to RCTs, which permits direct comparison between 2 diagnostic pathways with clinically relevant outcomes, as opposed to diagnostic cohort studies that can only inform us about test accuracy measures. Secondly, the implementation of Bayesian framework in network meta-analysis as well as in HSROC model could provide better confidence in terms of decision-making results, therefore it gives clarity for surgeons as 
well as patients for choosing the best strategy. However, it should also be taken into consideration that important limitation in our study was that the complications and cost outcome were not taken into account in the analysis of this study thus future research directions must address this specific analysis.

\section{Conclusion}

A key issue in the diagnosis and treatment of PCa extends in the need to identify PCa detection while still maintaining the clinically significant PCa detection rate that required intervention and to avoid the unnecessary diagnosis of low-risk, low-volume disease. Taken into consideration of four endpoints, this network meta-analysis and HSROC model highlight the important finding that FUS+TRUS-GB, FUS-GB, and MRI-GB showed their superiority in prostate cancer detection. Meanwhile, TRUS-GB had the lowest sensitivity and specificity therefore it was more associated with the harms of unnecessary biopsies and over-diagnosis. We hope that these results will assist in shared decision making between patients, carers, and their surgeons.

\section{List Of Abbreviations}

- TRUS-GB: Transrectal ultrasound-guided biopsy

- MRI-GB: Magnetic resonance imaging-guided biopsy

- MRI+TRUS (FUS)-GB: Fusion-guided biopsy

- FUS+TRUS-GB: Fusion + Transrectal ultrasound-guided biopsy

- TPUS-GB: Transperineal ultrasound-guided biopsy

- CEUS-GB: Contrast-enhanced ultrasound-guided biopsy

\section{Declarations}

- Ethical approval and consent to participate:

Not Applicable

- Consent for publication:

Not applicable

- Availability of supporting data:

The datasets used and/or analysed during the current study are available from the corresponding author on reasonable request. 
- Competing interests:

The authors declare that they have no competing interests

- Funding:

There is no funding of this study

- Author contributions

Ilham Akbar Rahman: study concept and design, data extraction, data analysis, data interpretation, drafted the manuscript, critical revision of the manuscript

Ilham Fauzan: Study concept and design, data extraction, data analysis, data interpretation, drafted the manuscript, critical revision of the manuscript

Syakri Syahrir: Study concept and design, data interpretation, critical revision of the manuscript

Harry Nusaly: Study concept and design, data interpretation, critical revision of the manuscript

Firdaus Kasim: design the model of analysis, data analysis, data interpretation, tables and figure design, critical revision of the manuscript

- Acknowledgements:

Not applicable

- Author's information:

Not applicable

\section{References}

1. Torre, L. A., Bray, F., Siegel, R. L., Ferlay, J., Lortet-Tieulent, J. \&, Jemal A. Global cancer statistics,2012. CA: A. Cancer J Clin. 2015;

2. Bray F., Ferlay J., Soerjomataram I., Siegel R.L., Torre L.A. JA. Global Cancer Statistics 2018: GLof Incidence and Mortality World in 185 Countries. CA Cancer J ClinAnticancer Res. 2018;

3. Xue J, Wang Y, Zheng Y, Zhang J, Qi F, Cheng H, et al. Efficacy characteristics of different therapeutic modalities for locally advanced prostate cancer: a Bayesian network meta-analysis of randomized controlled trials. Ann Transl Med. 2018;6(18):358-358.

4. Schröder FH, Hugosson J, Roobol MJ, Tammela TLJ, Ciatto S, Nelen V, et al. Screening and prostatecancer mortality in a randomized european study. N Engl J Med. 2009;

5. Elwenspoek MMC, Sheppard AL, Mclnnes MDF, Merriel SWD, Rowe EWJ, Bryant RJ, et al. Comparison of Multiparametric Magnetic Resonance Imaging and Targeted Biopsy with Systematic Biopsy Alone 
for the Diagnosis of Prostate Cancer: A Systematic Review and Meta-analysis. JAMA Netw Open. 2019;2(8):1-13.

6. Gustafsson O, Mansour E, Norming U, Carlsson A, Törnblom M, Nyman CR. Prostate-specific antigen (PSA), PSA density and age-adjusted PSA reference values in screening for prostate cancer: A study of a randomly selected population of 2,400 men. Scand J Urol Nephrol. 1998;

7. Waidelich R, Jansen HM, Stieber P, Schmeller N, Lamerz R, Werdan K, et al. Screening for prostatic carcinoma with prostate specific antigen. In: Anticancer Research. 1997.

8. Puech P, Rouvière O, Renard-Penna R, Villers A, Devos P, Colombel M, et al. Prostate cancer diagnosis: Multiparametric mr-targeted biopsy with cognitive and transrectal us-mr fusion guidance versus systematic biopsy-prospective multicenter study. Radiology. 2013;

9. Kitagawa Y, Namiki M. Prostate-specific antigen-based population screening for prostate cancer: Current status in Japan and future perspective in Asia. Asian Journal of Andrology. 2015.

10. Schröder FH. Prostate cancer around the world. An overview. Urologic Oncology: Seminars and Original Investigations. 2010.

11. Hussain M, Tangen CM, Berry DL, Higano CS, Crawford ED, Liu G, et al. Intermittent versus continuous androgen deprivation in prostate cancer. N Engl J Med. 2013;

12. Zhang $Q$, Cheng $H$, Wang $Y$, Tian $Y$, Xia J, Wang $Y$, et al. Different therapeutic regimens in the treatment of metastatic prostate cancer by performing a Bayesian network meta-analysis. Int J Surg [Internet]. 2019;66(September 2018):28-36. Available from:

https://doi.org/10.1016/j.ijsu.2019.04.006

13. Koh J, Jung DC, Oh YT, Yoo MG, Noh S, Han KH, et al. Additional targeted biopsy in clinically suspected prostate cancer: Prospective randomized comparison between contrast-enhanced ultrasound and sonoelastography guidance. Ultrasound Med Biol. 2015;

14. Caldwell DM, Ades AE, Higgins JPT. Simultaneous comparison of multiple treatments: Combining direct and indirect evidence. British Medical Journal. 2005.

15. Lu G, Ades AE. Combination of direct and indirect evidence in mixed treatment comparisons. Stat Med. 2004;

16. Trevethan R. Sensitivity, Specificity, and Predictive Values: Foundations, Pliabilities, and Pitfalls in Research and Practice. Front Public Heal. 2017;

17. Parikh R, Mathai A, Parikh S, Sekhar GC, Thomas R. Understanding and using sensitivity, specificity and predictive values. Indian J Ophthalmol. 2008;

18. Egger M, Davey Smith G, Schneider M, Minder C. Bias in meta-analysis detected by a simple, graphical test BMJ 315: 629-634. Find this Artic online. 1997;

19. Rouse B, Chaimani A, Li T. Network meta-analysis: an introduction for clinicians. Intern Emerg Med. 2017;

20. Rastinehad AR, Turkbey B, Salami SS, Yaskiv O, George AK, Fakhoury M, et al. Improving detection of clinically significant prostate cancer: Magnetic resonance imaging/transrectal ultrasound fusion 
guided prostate biopsy. J Urol [Internet]. 2014;191(6):1749-54. Available from:

http://dx.doi.org/10.1016/j.juro.2013.12.007

21. Alberts AR, Schoots IG, Bokhorst LP, Drost FJH, van Leenders GJ, Krestin GP, et al. Characteristics of Prostate Cancer Found at Fifth Screening in the European Randomized Study of Screening for Prostate Cancer Rotterdam: Can We Selectively Detect High-grade Prostate Cancer with Upfront Multivariable Risk Stratification and Magnetic Reson. Eur Urol. 2018;73(3):343-50.

22. Taverna G, Bozzini G, Grizzi F, Seveso M, Mandressi A, Balzarini L, et al. Endorectal multiparametric 3tesla magnetic resonance imaging associated with systematic cognitive biopsies does not increase prostate cancer detection rate: a randomized prospective trial. World J Urol. 2016;34(6):797-803.

23. Kim JW, Lee HY, Hong SJ, Chung BH. Prospective Trial 612 cores Korea.pdf. Vol. 45, Yonsei Medical Journal. 2004. p. 671-5.

24. de la Rosette JJMCH, Wink MH, Mamoulakis C, Wondergem N, ten Kate FJC, Zwinderman K, et al. Optimizing Prostate Cancer Detection: 8 Versus 12-Core Biopsy Protocol. J Urol [Internet]. 2009;182(4 SUPPL.):1329-36. Available from: http://dx.doi.org/10.1016/j.juro.2009.06.037

25. Taymoorian K, Thomas A, Slowinski T, Khiabanchian M, Stephan C, Lein M, et al. Transrectal broadband-Doppler sonography with intravenous contrast medium administration for prostate imaging and biopsy in men with an elevated PSA value and previous negative biopsies. Anticancer Res. 2007;27(6 C):4315-20.

26. Rochester MA, Griffin S, Chappell B, McLoughlin J. A prospective randomised trial of extended core prostate biopsy protocols Utilizing 12 versus 15 cores. Urol Int. 2009;83(2):155-9.

27. Ghafoori M, Velayati M, Ghasabeh MA, Shakiba M, Alavi M. Prostate biopsy using transrectal ultrasonography; the optimal number of cores regarding cancer detection rate and complications. Iran J Radiol. 2015;12(2):0-4.

28. Mitterberger M, Horninger W, Pelzer A, Strasser H, Bartsch G, Moser P, et al. A prospective randomized trial comparing contrast-enhanced targeted versus systematic ultrasound guided biopsies: Impact on prostate cancer detection. Prostate. 2007;

29. Emiliozzi P, Corsetti A, Tassi B, Federico G, Martini M, Pansadoro V. Best approach for prostate cancer detection: A prospective study on transperineal versus transrectal six-core prostate biopsy. Urology. 2003;61(5):961-6.

30. Paul R, Schöler S, Van Randenborgh H, Kübler H, Alschibaja M, Busch R, et al. Optimization of prostatic biopsy: A prospective randomized trial comparing the sextant biopsy with a 10-core biopsyImpact of prostatic region of sampling. Urol Int. 2005;74(3):203-8.

31. Hara R, Jo Y, Fujii T, Kondo N, Yokoyoma T, Miyaji Y, et al. Optimal Approach for Prostate Cancer Detection as Initial Biopsy: Prospective Randomized Study Comparing Transperineal Versus Transrectal Systematic 12-Core Biopsy. Urology. 2008;71(2):191-5.

32. Arsov C, Rabenalt R, Blondin D, Quentin M, Hiester A, Godehardt E, et al. Prospective randomized trial comparing magnetic resonance imaging (MRI)-guided in-bore biopsy to MRI-ultrasound fusion and 
transrectal ultrasound-guided prostate biopsy in patients with prior negative biopsies. Eur Urol. 2015;68(4):713-20.

33. Shoji S, Hiraiwa S, Ogawa T, Kawakami M, Nakano M, Hashida K, et al. Accuracy of real-time magnetic resonance imaging-transrectal ultrasound fusion image-guided transperineal target biopsy with needle tracking with a mechanical position-encoded stepper in detecting significant prostate cancer in biopsy-naïve men. Int J Urol. 2017;24(4):288-94.

34. Tewes S, Peters I, Tiemeyer A, Peperhove M, Hartung D, Pertschy S, et al. Evaluation of MRI/Ultrasound Fusion-Guided Prostate Biopsy Using Transrectal and Transperineal Approaches. Biomed Res Int. 2017;2017.

35. Kasivisvanathan V, Rannikko AS, Borghi M, Panebianco V, Mynderse LA, Vaarala MH, et al. MRItargeted or standard biopsy for prostate-cancer diagnosis. N Engl J Med. 2018;378(19):1767-77.

36. Choi YH, Kang MY, Sung HH, Jeon HG, Chang Jeong B, Seo S II, et al. Comparison of Cancer Detection Rates Between TRUS-Guided Biopsy and MRI-Targeted Biopsy According to PSA Level in Biopsy-Naive Patients: A Propensity Score Matching Analysis. Clin Genitourin Cancer [Internet]. 2019;17(1):e19-25. Available from: https://doi.org/10.1016/j.clgc.2018.09.007

37. Baco E, Rud E, Eri LM, Moen G, Vlatkovic L, Svindland A, et al. A Randomized Controlled Trial to Assess and Compare the Outcomes of Two-core Prostate Biopsy Guided by Fused Magnetic Resonance and Transrectal Ultrasound Images and Traditional 12-core Systematic Biopsy. Eur Urol [Internet]. 2016;69(1):149-56. Available from: http://dx.doi.org/10.1016/j.eururo.2015.03.041

38. Halpern EJ, Ramey JR, Strup SE, Frauscher F, McCue P, Gomella LO. Detection of prostate carcinoma with contrast-enhanced sonography using intermittent harmonic imaging. Cancer. 2005;104(11):2373-83.

39. Delgado Oliva F, Arlandis Guzman S, Bonillo García M, Broseta Rico E, Boronat Tormo F. Diagnostic performance of power doppler and ultrasound contrast agents in early imaging-based diagnosis of organ-confined prostate cancer: Is it possible to spare cores with contrast-guided biopsy? Eur $\mathrm{J}$ Radiol [Internet]. 2016;85(10):1778-85. Available from: http://dx.doi.org/10.1016/j.ejrad.2016.07.021

40. Porpiglia F, Manfredi M, Mele F, Cossu M, Bollito E, Veltri A, et al. Diagnostic Pathway with Multiparametric Magnetic Resonance Imaging Versus Standard Pathway: Results from a Randomized Prospective Study in Biopsy-naïve Patients with Suspected Prostate Cancer. Eur Urol [Internet]. 2017;72(2):282-8. Available from: http://dx.doi.org/10.1016/j.eururo.2016.08.041

41. Rodríguez-Covarrubias F, González-Ramírez A, Aguilar-Davidov B, Castillejos-Molina R, Sotomayor M, Feria-Bernal G. Extended sampling at first biopsy improves cancer detection rate: Results of a prospective, randomized trial comparing 12 versus 18-core prostate biopsy. J Urol [Internet]. 2011;185(6):2132-6. Available from: http://dx.doi.org/10.1016/j.juro.2011.02.010

42. Sonn GA, Chang E, Natarajan S, Margolis DJ, MacAiran M, Lieu P, et al. Value of targeted prostate biopsy using magnetic resonance-ultrasound fusion in men with prior negative biopsy and elevated prostate-specific antigen. Eur Urol. 2014; 
43. Taverna G, Morandi G, Seveso M, Giusti G, Benetti A, Colombo P, et al. Colour Doppler and microbubble contrast agent ultrasonography do not improve cancer detection rate in transrectal systematic prostate biopsy sampling. BJU Int. 2011;108(11):1723-7.

44. Rutter CM, Gatsonis CA. A hierarchical regression approach to meta-analysis of diagnostic test accuracy evaluations. Stat Med. 2001;

45. Loeb S, Bjurlin MA, Nicholson J, Tammela TL, Penson DF, Carter HB, et al. Overdiagnosis and overtreatment of prostate cancer. Eur Urol. 2014;65(6):1046-55.

46. Spiegelhalter DJ. Incorporating Bayesian ideas into health-care evaluation. Stat Sci. 2004;19(1):15674.

47. O’Hagan A. Bayesian statistics: principles and benefits. Bayesian Stat Qual Model agro-food Prod Chain (Wageningen UR Front Ser. 2004;31-45.

48. Grenabo Bergdahl A, Wilderäng U, Aus G, Carlsson S, Damber JE, Frånlund $M$, et al. Role of Magnetic Resonance Imaging in Prostate Cancer Screening: A Pilot Study Within the Göteborg Randomised Screening Trial. Eur Urol. 2016;

49. Terris MK, Hammerer PG, Nickas ME. Comparison of ultrasound imaging in patients undergoing transperineal and transrectal prostate ultrasound. Urology. 1998;52(6):1070-2.

50. Halpern El, Frauscher F, Strup SE, Nazarian LN, O’Kane P, Gomella LG. Prostate: High-frequency doppler US imaging for cancer detection. Radiology. 2002;225(1):71-7.

51. Ahmed HU, Kirkham A, Arya M, Illing R, Freeman A, Allen C, et al. Is it time to consider a role for MRI before prostate biopsy? Nat Rev Clin Oncol. 2009;6(4):197-206.

52. Carlsson S V., de Carvalho TM, Roobol MJ, Hugosson J, Auvinen A, Kwiatkowski M, et al. Estimating the harms and benefits of prostate cancer screening as used in common practice versus recommended good practice: A microsimulation screening analysis. Cancer. 2016;122(21):3386-93.

53. Heijnsdijk EAM, De Carvalho TMD, Auvinen A, Zappa M, Nelen V, Kwiatkowski M, et al. Costeffectiveness of prostate cancer screening: A simulation study based on ERSPC data. J Natl Cancer Inst. 2015;107(1):1-9.

\section{Tables}

Table 1. PICO of the study

\begin{tabular}{|l|l|}
\hline Patient & Patient with clinical suspicion of prostate cancer (high PSA or abnormal DRE) \\
\hline Intervention & TRUS-GB $(6,8,10,12,15,18$ cores), TPUS-GB, CEUS-GB, FUS-GB, MRI-GB, FUS+TRUS-GB \\
\hline Control & TRUS-GB $(6,8,10,12,15,18$ cores), TPUS-GB, CEUS-GB, FUS-GB, MRI-GB, FUS+TRUS-GB \\
\hline Outcome & $\begin{array}{l}\text { Overall prostate cancer, clinically significant, insignificant detection rate, sensitivity and } \\
\text { specificity }\end{array}$ \\
\hline
\end{tabular}

Table 2. Baseline characteristics of selected studies

[Please see the supplementary files section to view Table 2.] 
Table 3: Detailed rank probability based on Surface Under Cumulative Rank (SUCRA). (A) Rank probability for PCa detection rate; (B) Rank probability for significant PCa detection rate; (C) Rank probability for insignificant PCa detection rate.

[Please see the supplementary files section to view Table 3.]

\section{Figures}

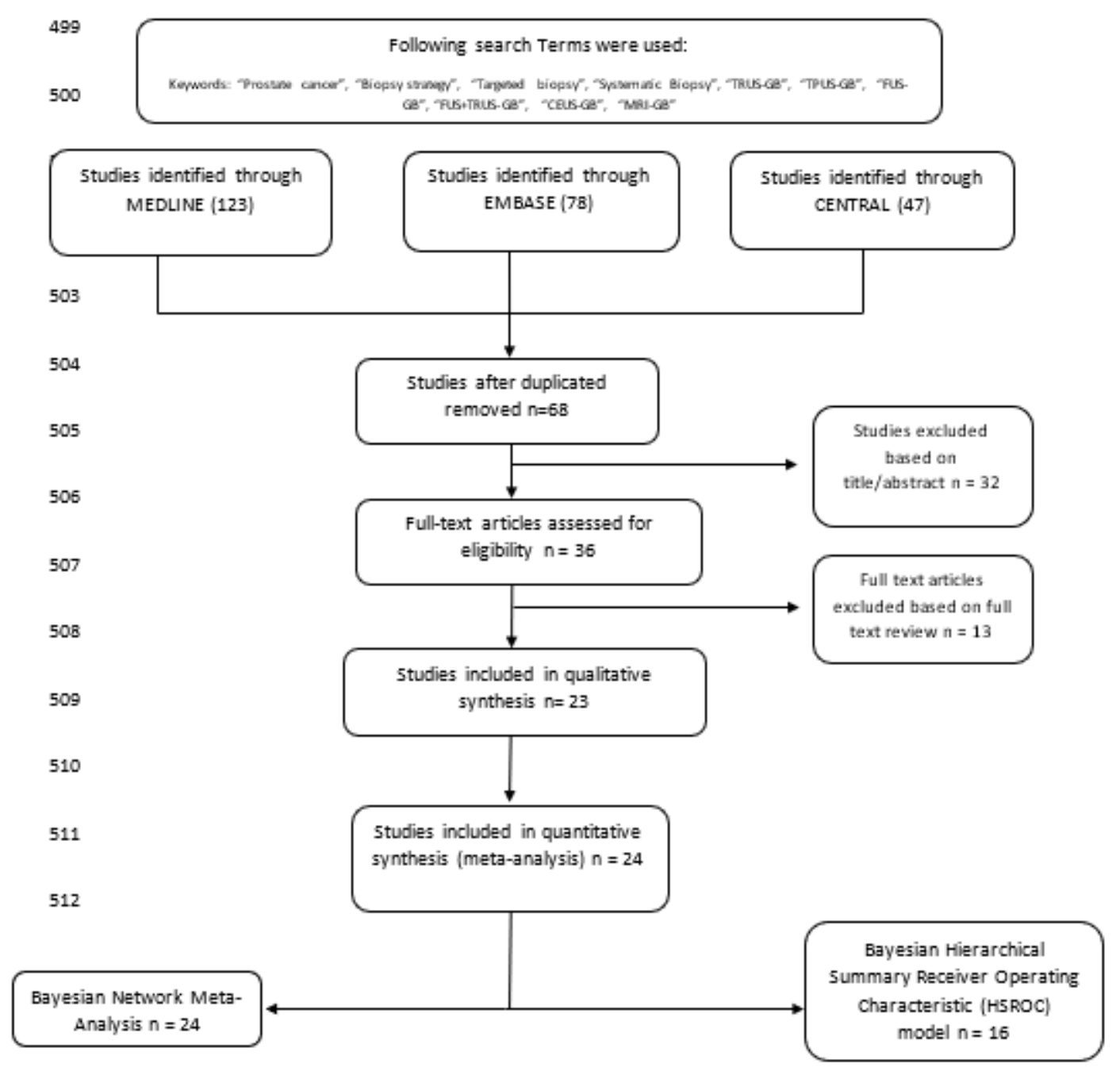

\section{Figure 1}

Flow chart of systematic literature identification and selection process implementing PRISMA (Preferred Reporting Items for Systematic Review and Meta-Analysis) Guide 

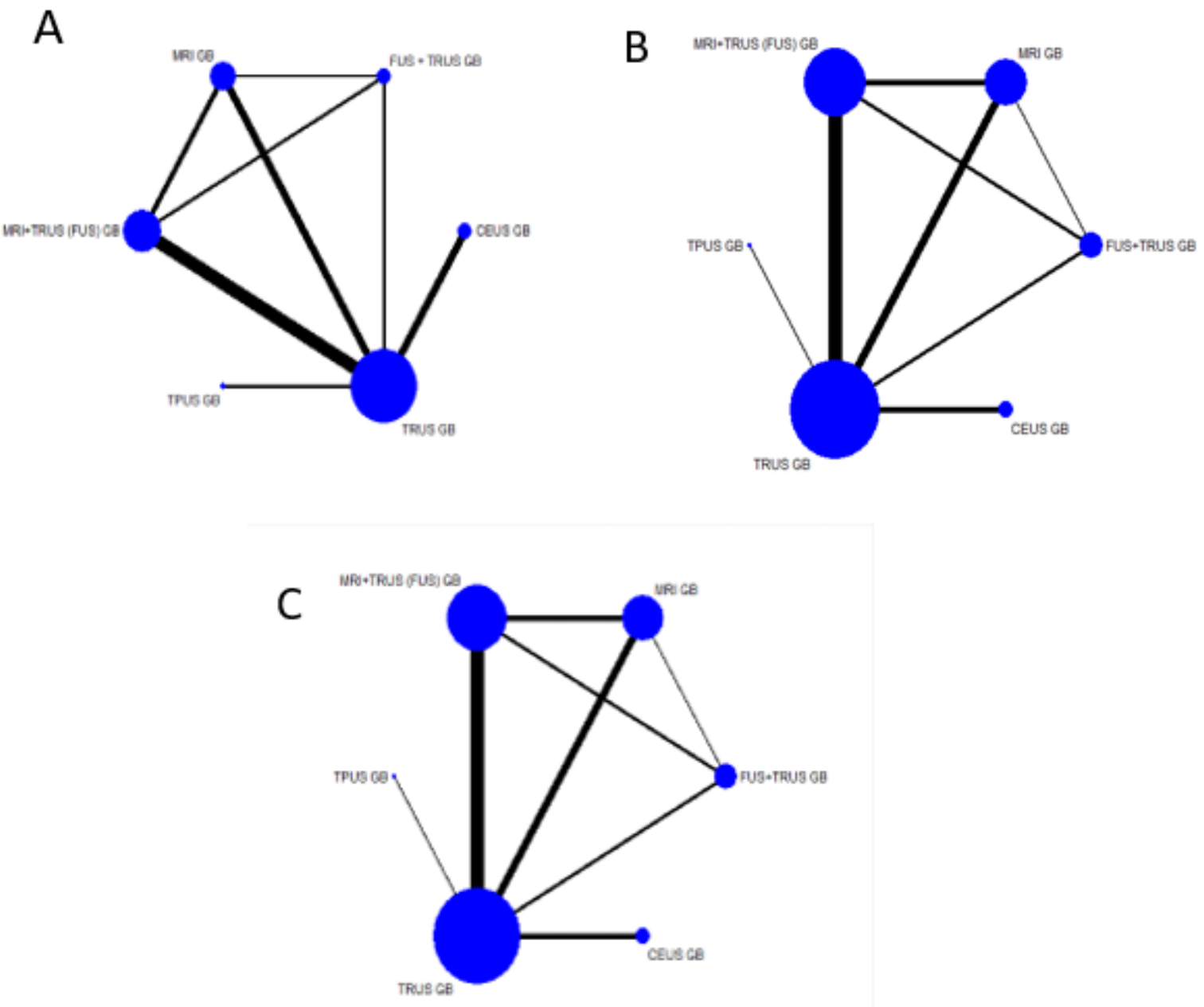

\section{Figure 3}

Network comparison structure diagrams of different biopsy strategies. (A) PCa detection rate; (B) Clinically significant PCa detection rate; (C) Insignificant PCa detection rate. The size of every circle is proportional to the number of randomly assigned patients and indicates the sample size. The thickness of the line corresponds to the number of trials. Direct comparisons are linked with I 

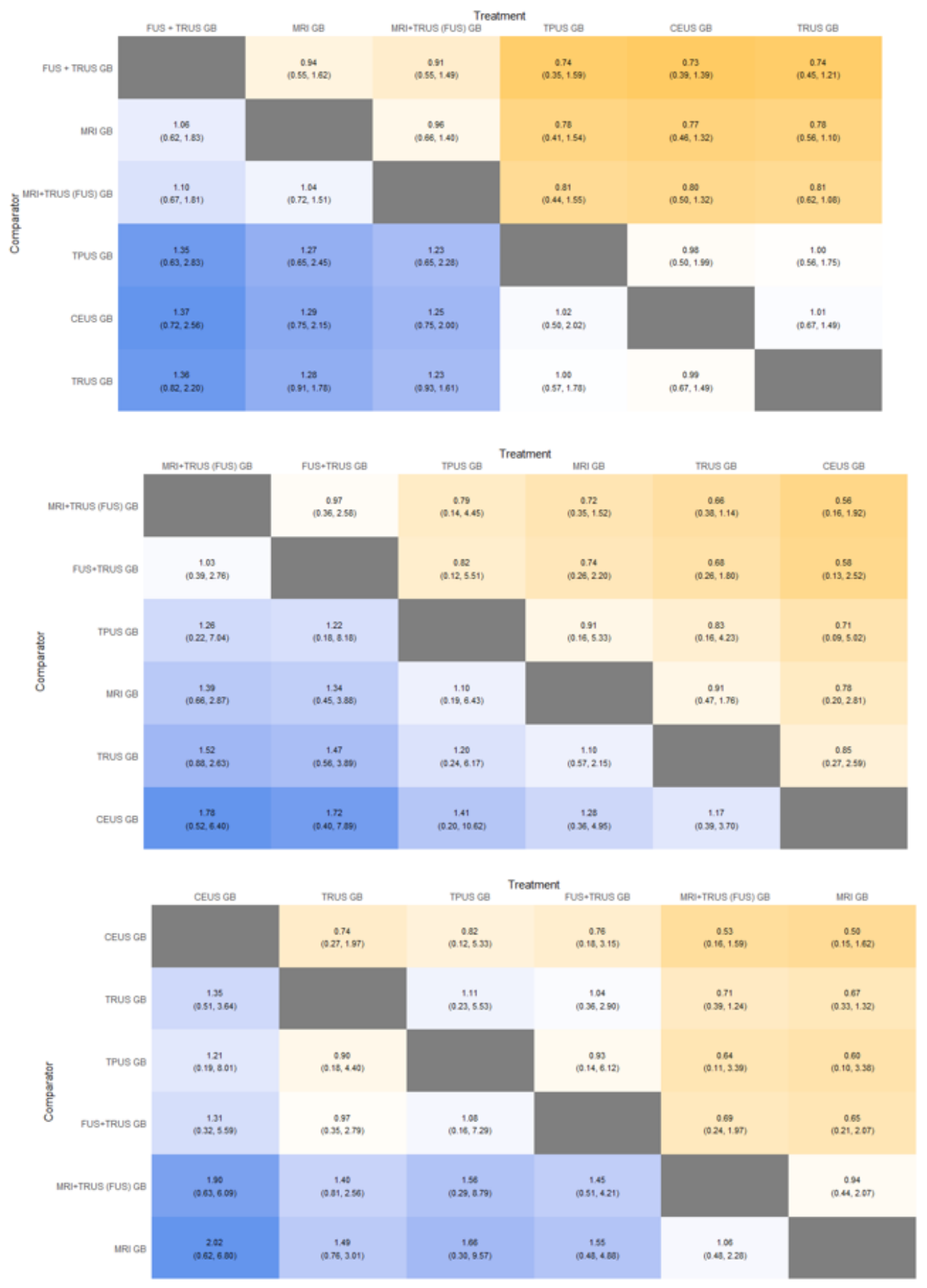

\section{Figure 5}

Network Meta-Analysis using odds ratio with $95 \%$ credible intravals of different biopsy strategies. A blue cell indicates that a treatment performed better than its comparator (estimate greater than 1), while an orange cells indicates that the treatment performed worse than its comparator (estimate smaller than 1).. The strategy has been sorted from left to right according to SUCRA ranking as from worst to best 
respectively. (A) Overall PCa outcome detection rate outcome, (B) Clinically significant PCa detection rate outcome, (C) Insignificant PCa detection rate outcome
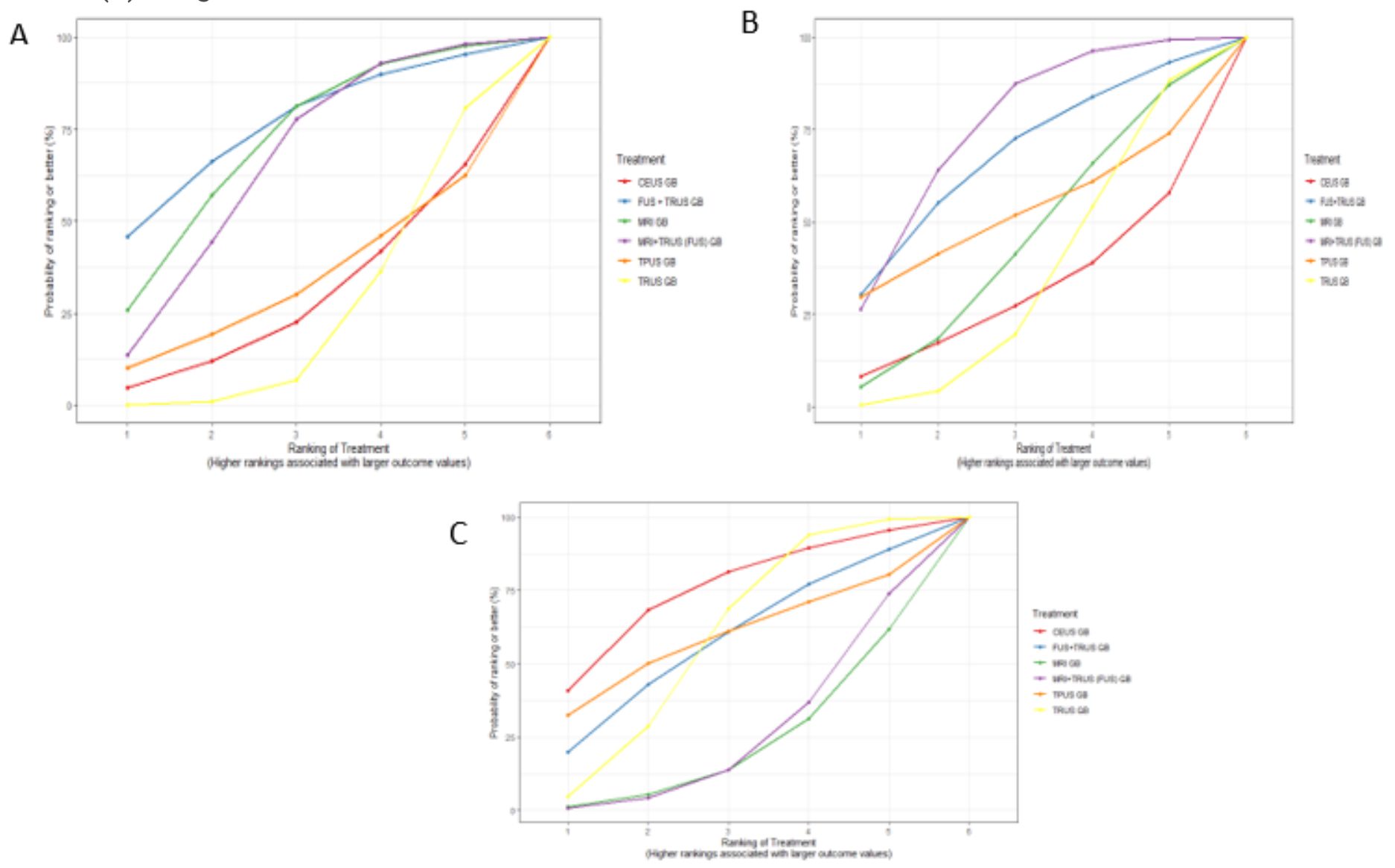

\section{Figure 7}

SUCRA plot for clinical outcomes. (A) Overall PCa detection rate; (B) Clinically significant PCa detection; (C) Insignificant PCa detection. 

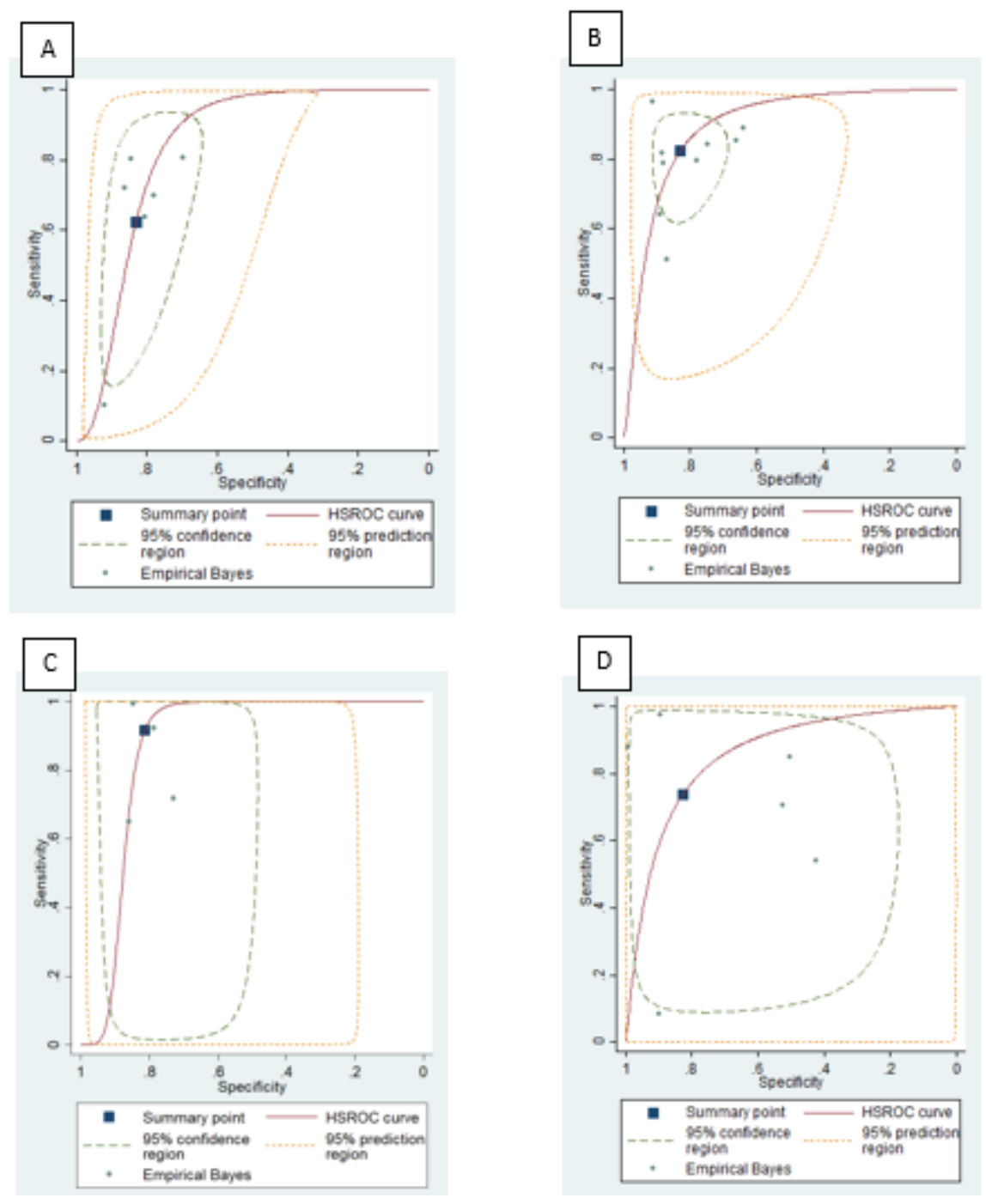

Figure 9

Bayesian prediction served in Hierarchical Summary Receiver Operating Characteristic (HSROC) model by Rutter and Gatsonis for prediction of true sensitivity and specificity of (A) TRUS-GB; (B) MRI+TRUS (FUS) GB; (C) MRI-GB; (D) CEUS-GB

\section{Supplementary Files}

This is a list of supplementary files associated with this preprint. Click to download.

- Table3.docx

- Table2.docx

- Table3.docx

- Table2.docx 